\title{
A New Dynamic 3-D Virtual Methodology for Teaching the Mechanics of Atrial Septation as seen in the Human Heart
}

\section{Authors}

Jean-Marc Schleich ${ }^{1,2,3}$, Jean-Louis Dillenseger ${ }^{2,3}$, Lucile Houyel ${ }^{4}$, Claude Almange ${ }^{1}$, Robert H. Anderson ${ }^{5}$

\section{Institutions}

${ }^{1}$ Département de Cardiologie et Maladies Vasculaires, Hôpital de Pontchaillou, CHU Rennes, France.

${ }^{2}$ INSERM, U642, Rennes, F-35000, France;

${ }^{3}$ Université de Rennes 1, LTSI, Rennes, F-35000, France;

${ }^{4}$ Service de Chirurgie Cardiaque Pédiatrique, Hôpital Marie-Lannelongue, 92350 Le Plessis Robinson, France

${ }^{5}$ Cardiac Unit. Institute of Child Health. University College London. 30 Guilford Street. London WC1N 1EH, UK

\section{Corresponding Author}

Jean-Marc Schleich, M.D, PhD.

Département de Cardiologie et Maladies Vasculaires, Hôpital de Pontchaillou, 35033 Rennes cédex, France.

Phone : $33+(0) 299282517$

Fax : $33+(0) 299282518$

Email: jean-marc.schleich@ chu-rennes.fr 


\begin{abstract}
Background: Learning embryology remains difficult, since it requires understanding of many complex phenomena. The temporal evolution of developmental events has classically been illustrated using cartoons, which create difficulty in linking spatial and temporal aspects, such correlation being the keystone of descriptive embryology.

Methods: We synthesized the bibliographic data from recent studies of atrial septal development. On the basis of this synthesis, consensus on the stages of atrial septation as seen in the human heart has been reached by a group of experts in cardiac embryology and paediatric cardiology. This has permitted the preparation of three-dimensional (3D) computer graphic objects for the anatomical components involved in the different stages of normal human atrial septation.

Results: We have provided a virtual guide to the process of normal atrial septation, the animation providing an appreciation of the temporal and morphologic events necessary to separate the systemic and pulmonary venous returns.
\end{abstract}

Conclusion: We have shown that our animations of normal human atrial septation increase significantly the teaching of the complex developmental processes involved, and provide a new dynamic for the process of learning.

Key words: Embryology; atrial septation; computer graphics; Medical education 


\section{INTRODUCTION}

It is axiomatic that accurate knowledge of normal cardiac development is essential for properly understanding the morphogenesis of congenital cardiac malformations. Appreciation of the events occurring during cardiac development, however, remains difficult. It requires a clear perception of the appearance, spatial location, and function of a number of complex anatomical structures. This information is usually provided by sketches or drawings, which are designed to facilitate mental visualization, and hence understanding, of the spatiotemporal evolution of the developmental sequence (Harvey and Rosenthal, 1999; Houyel, 1998; Schoenwolf et al., 2008). Such drawings and sketches, nonetheless, may not be the most effective means of communication, since cartoons prepared of necessity in two dimensions give only a partial impression of the spatial geometry of the objects illustrated. Moreover, the dynamics of structural evolution are rendered in less than complete fashion by a limited sequence of static images. This inadequacy in presentation of complex processes of spatial development is a major limitation to understanding, with significant efforts required graphically to illustrate the temporal sequence of development of most cardiac structures.

Use of advanced computer graphics has now been shown to provide substantial improvements for medical teaching in general (Dev, 1999; Higgins et al., 1998), and cardiac morphogenesis in particular (Laan et al., 1989; Whiten et al., 1998). We have found that computer graphics provide solutions for the design, modelling, visualization, and manipulation of threedimensional (3-D) objects, as well as for generating animated film sequences (Berlage, 1998). With such techniques, it is now possible to show evolutions of events that were previously inaccessible (Schleich and Almange, 2000). This dynamic aspect is the cornerstone of descriptive embryology. Our current aim, therefore, was to create teaching material in the form of an animated sequence of virtual images, along with a commentary, so as to improve the understanding of the complex events occurring during septation of the atrial chambers in 
the human heart. We describe the construction of a sequence of virtual images, explaining the options taken during the design of our model, and the presentation of the results. We also describe the formal evaluation undertaken to validate our opinion that such use of virtual animations can improve substantially the understanding of the complex events occurring during normal atrial septation.

\section{CONSTRUCTION OF THE MODEL}

Our overall objective was to design and develop a 3-D model showing the dynamics of the various stages of normal development and septation of the atrial chambers in the human heart. Such embryological modelling is achieved conventionally through reconstruction of serially sectioned murine hearts (Brune et al., 1999; Dhenain et al., 2001). The mouse heart, however, is unsuitable to use as a model of human development. This is because the pulmonary vein remains a solitary channel as it enters the left atrium in the mouse, while the left superior caval vein remains as a patent channel. The structure of the oval foramen is also markedly different in the mouse compared to man (Webb et al., 1996). Moreover, reconstructions of serially sectioned embryos provide no more than a view at fixed periods, losing the continuity required over time. Although we have access to serially sectioned human hearts, as yet we have not developed the facilities to reconstruct these sections. Based on examination of this material, however, along with study of previous descriptions, we chose to model the developing heart, rather than reconstructing it. The work required close cooperation between paediatric cardiologists and the graphic modeller in charge of designing the three-dimensional animated sequence. We created, therefore, a pattern of development to integrate our anatomic knowledge into the final product. This pattern can be summarized as follows:

\section{Creation of the scene}

In a first step, the current state of understanding of atrial septal development was reviewed by a multidisciplinary group of physicians, including cardiologists, paediatric cardiologists, and 
morphologists. The approach took into account early descriptions, forgotten subsequent to their publication, such as the account given by His for formation of the vestibular spine (His, 1880), and the study by Röse which described the infolding of the superior rim of the oval fossa (Röse, 1899). These important studies themselves point to the deficiencies in accounts of atrial septation as they appear in currently popular textbooks of embryology, such as the one edited by Larsen (Larsen, 1993) and renewed by Schoenwolf et al. (Schoenwolf et al., 2008). The group also took note of more recent studies correlating studies of development with knowledge of the morphology of the atrial septum in the human (Anderson et al., 2002; Anderson et al., 2003; Kim et al., 2001; Wessels et al., 2000).

\section{Development of the atrial septum in the human heart}

Septation of the atrial component of the primary heart tube begins at the end of the $4^{\text {th }}$ week of gestation, and continues well into the $3^{\text {rd }}$ month. During that time, the embryo grows from 4 to $6 \mathrm{~mm}$ to around $50 \mathrm{~mm}$ in crown-rump length. Concomitant with the septation of the atrial chambers, the apical parts of the ventricles balloon in sequence from the ventricular loop, the muscular ventricular septum appearing between them in consequence of the ballooning.

The first evidence of septation of the developing atrial component of the heart tube comes with the appearance of the primary atrial septum. This develops as a crescentic muscular partition, growing from the upper part of the atrial component of the primary heart tube, and taking its origin to the left of the right pulmonary ridge, this being the rightward margin of the persisting dorsal mesocardium. By the time of appearance of the primary septum from the atrial roof, the systemic venous tributaries have already remoulded from being bilaterally symmetrical to opening exclusively to the right side of the atrial component. Only subsequent to this rightward shift do the boundaries of the systemic venous component become evident as the venous valves, and only subsequent to the appearance of these valves is it possible to recognise a discrete systemic venous sinus (Anderson et al., 2006). By this stage, the pulmonary vein has also canalised as a solitary channel, which opens into the left side of the 
atrial component of the heart tube adjacent to the left side of the atrioventricular junction (Figure 1). As the primary atrial septum increases in size, it grows towards the atrioventricular endocardial cushions, themselves approaching each other so as to divide the atrioventricular canal (Figure 1). As the primary atrial septum grows towards the cushions, passing between the systemic venous sinus and the opening of the solitary pulmonary vein, it carries on its leading edge a prominent cap of mesenchymal tissue (Figure 2). This mesenchymal cap fuses initially with the superior atrioventricular cushion. At the same time, a protrusion of mesenchymal tissue from the dorsal mesocardium expands through the right pulmonary ridge. This structure, initially described by His as the "spina vestibuli" (Figure 4), and now termed the vestibular spine, grows towards the inferior atrioventricular cushion (Figure 3), reinforcing the area of attachment of the primary atrial septum to the superior cushion. During this growth of the primary atrial septum, and the appearance of the vestibular spine, the superior and inferior atrioventricular cushions have themselves fused, dividing the atrioventricular canal into the presumptive mitral and tricuspid valvar orifices.

Prior to fusion of the various mesenchymal components, there is a communication distal to the leading edge of the primary atrial septum. This is the primary interatrial foramen, or "ostium primum" (Figure 2). Before this hole is closed, multiple fenestrations appear in the cranial part of the primary septum. Coalescence of these fenestrations produces the secondary interatrial foramen, or "ostium secundum". At this stage, the cranial margin of this second foramen is no more than the roof of the atrial chamber (Figure 3). Subsequent to formation of the secondary interatrial foramen, the mesenchymal components all fuse together to close the primary foramen. Evidence from studies in the developing mouse heart show that dorsal mesocardial protrusion then muscularises to form the plump antero-inferior rim of the oval foramen (Snarr et al., 2008; Snarr et al., 2007a; Snarr et al., 2007b). It is only with subsequent migration of the orifice of the pulmonary vein from a position close to the atrioventricular junction to its final position in the roof of the left atrium, with concomitant appearance of four pulmonary 
venous orifices, that the deep infolding in the roof of the atrium typically described as the "septum secundum" becomes evident (Figure 5). In reality, this partition between the right and left atrial chambers is a deep groove between the orifices of the caval veins and the right pulmonary veins, as described long since by Röse (Röse, 1899). Subsequent to birth, it is this fold that provides the buttress against which the flap valve, representing the primary atrial septum, abuts to close the oval foramen (Figure 6).

\section{Proposal for Sequence of Shapes}

From the developmental sequence described above, which correlates well with the structure of the definitive atrial septum in the human heart (Figure 6), the experts defined several development steps involving the main structures necessary for atrial septation, namely the primary atrial septum, its mesenchymal cap, the atrioventricular endocardial cushions, the vestibular spine, and the superior interatrial fold. The experts also discussed the changes in shape occurring with the presumed sequence of atrial development.

The modeller then produced a storyboard to define the origins, forms, and dimensions between the different elements involved in atrial septation (Figure 7). The proposed storyboard was submitted to the medical experts for validation, and was modified as deemed necessary. When the proposed three-dimensional shapes were considered too complex to be represented in two dimensions, plaster models were constructed to improve the understanding of the spatial relationships of the different structures involved in the process of septation. Once the modifications were agreed and validated, further details were added to elucidate the shapes, spatial extension, and the relationship in space of the main structures during the temporal evolution of septation. A new storyboard was then generated and reviewed again by the experts. It was these final static shapes that formed the final storyboard.

\section{3-D Modelling}

The three-dimensional sequence was designed by the graphic modeller, and reviewed by the group of experts. On the basis of the storyboard, the overall sequence was organized around a 
limited number of static three-dimensional, representing the agreed crucial stages of atrial development. The modelling process then took place as follows (Schleich et al., 2002):

- Modelling of the agreed shapes. Having agreed the basic patterns, the modeller used control points to produce a surface model, assigning basic colours to the various structures, choosing relatively unsophisticated surface attributes. The shapes generated were submitted to the medical experts for validation.

- Animations to link the shapes. Animation was produced by point-to-point linear morphing from one main shape to another (Figure 8). The shapes at the beginning and end of the animation were modelled in parallel to achieve a point-to-point correspondence. In instances of significant deformation, intermediate shapes were produced to smooth the morphing.

The group of medical experts evaluated the animations on a regular basis, feeding back potential modifications, such as the speed of development of one element of the septal complex compared to the others. It also proved necessary to incorporate new developments, such as the appearance of the secondary foramen.

Once the three-dimensional sequence was complete, it was refined with surface attributes, such as colour, texture, and shadows, so as to produce a realistic end product. Colours were selected to appear as natural as possible, or to follow conventional rules. The realistic aspect was also enhanced by used of textures extracted from medical images, such as photographs of operative procedures (Figure 9).

We then created a film showing the dynamics of atrial septation, using Newtek's LightWave ${ }^{\mathrm{TM}}$ product (Newtek, San Antonio, TX, USA). The final animation was edited using Adobe Premiere software. Oral commentary was added, explaining the main steps of development. Arrows were added when necessary to pinpoint certain specific anatomical changes. 
The final animation and commentary are available in AVI and mpeg video format, which can be displayed on any standard Personal Computer using an interactive CD or DVD. This animation can also be viewed on a videotape (in PAL or NTSC format) or DVD-Video. These latter options may be better suited for demonstrating normal atrial septation in the lecture room.

\section{EVALUATION}

We took several considerations into account when seeking to evaluate the performance of our developed multimedia project (Achtemeier et al., 2003; Huang, 2005). We acknowledged that it was necessary to evaluate first the effectiveness of its usability, and second the outcomes in terms of learning. Since the answers to both points are subjective, evaluation has to be performed by questioning attitudinal and open responses. Our questionnaire designed to take note of both aspects is shown in the appendix. We measured attitudinal responses using a 5 point Lickert rating scale from strongly disagree to strongly agree.

We have distributed the animations to several institutions worldwide, sending 34 evaluations to those studying the animations, and obtaining 25 responses. We were able to draw several inferences from the answers received:

\section{Use of tool.}

Most of the users made moderate use of the tool, usually from 2 to 5 times each year, albeit 4 responders used the product more than 5 times each year. The animation was used for teaching small groups on 15 occasions, for main courses in classrooms in 9 instances, and for self-learning in 5 . In 8 instances, the tool was also used by the instructor for personal education.

\section{Effective usability}


Except for one neutral answer, all agreed that navigation through the modules was easy, a response confirmed by the open answers. Some responders, nonetheless, suggested that a learning phase was needed, while others suggested improvements, especially for navigating within the animations. Only one of our responders encountered technical problems produced by the operating system.

\section{Learning outcomes}

All agreed that the animation was useful for understanding the mechanics of atrial septation, with more than half agreeing strongly. All also agreed, or strongly agreed, that the module helped conceptual understanding. We received open comments such as "very didactic", "good balance between simplification and realism", "really useful for the spatial and temporal understanding", "appropriate for summarizing", and "stimulated the motivation of the students for learning”. Feedback from the instructors was also very encouraging, especially regarding the use of the sequences during main courses.

\section{DISCUSSION}

Most publications on three-dimensional reconstruction have essentially focused on constituting databases (Ringwald et al., 1994). Such reconstructions have originated either from serial sections obtained using optical microscopy (Brune et al., 1999; Laan et al., 1989; Whiten et al., 1998), from electron microscopic images (Wenink and Chon, 1984), or more recently from data acquired using magnetic resonance imaging (Dhenain et al., 2001), optical projection tomography (Sharpe et al., 2002), or episcopic fluorescence imaging (Weninger and Mohun, 2002). Such studies have improved the understanding of cardiac structure at a given time of development, but representing static points in time, have of necessity not provided the dynamics required to appreciate the temporal sequence of cardiac development. The use of virtual images permits three-dimensional modelling of all steps of cardiac embryology, and the creation of dynamic sequences. Moreover, when dealing with the early stages of the 
cardiac development, the techniques discussed above have thus far been performed only on experimental animals, producing results which are less than ideal when considering atrial septation in the human heart. To our knowledge, our study is the first attempt to use techniques of virtual imaging to elucidate the development of the atrial septum in the human heart. Morphing techniques, which display events in four dimensions, are perfectly adapted to this specific type of teaching. Using commercial graphics software, other teams can involve themselves in embryology, as well as anatomy, physiology, and so on. Moreover, as we have the 3D shapes, they could be manipulated by a 3D viewer.

This animation has been used in the University in Rennes for several courses on atrial development, and this experience has demonstrated that virtual imaging significantly improves the understanding of complex phenomena. To validate this opinion, we designed a questionnaire to compare the classical and dynamic approaches. The assessment of the teachers themselves, combined with the remarks and comments of the students, showed that this type of animation aided significantly in the understanding of normal atrial septation, both spatially and temporally. Another positive aspect is that feedback suggested that memorizing the overall mechanisms of atrial septation became much easier compared to classical lessons based on cartoons. Animation, nonetheless, has a major drawback if used in isolation. The attention of the students is focused mainly on the animation itself, in other words on the spatio-temporal aspects of the overall events involved in septation. Precise anatomical details, such as the names and functions of the various developmental components, are usually not simultaneously integrated during visualization of the sequence, and must be learnt afterwards. This type of animation, therefore, is an aid to understanding, but still requires learning the mechanics of atrial septation. 
A few errors or inaccuracies may have entered the creation of the 3-D objects and/or 3-D morphing scenes, but the ultimate purpose of the animation was to show that it is possible, with virtual images, to improve training for the dissemination of the steps involved in cardiac septation. The virtual nature of the animation makes it amenable to change should new data appear, or if the experts deem that modifications are needed. Moreover, rigorously exact modelling would have been counterproductive when judged against our aim to facilitate the understanding of elementary embryological mechanisms based on correlations with the definitive atrial structures. We elected to simplify the situation so as to produce a model that would be readily accessible to students. It is now our intention to integrate the animation and three-dimensional objects into a computed-aided teaching tool, which will include exhaustive explanatory texts, interactive commentaries, and a set of bibliographic references. It is our hope that this tool can be expanded to help self-learning of overall normal cardiac development. With this in mind, we are now exploring ways of applying new technologies to higher education.

\section{Acknowledgments}

We thank Stéphane Andru, who designed the three-dimensional objects and the animated sequence, and Pierre Galluc for the development of the Open GL 3-D viewer. 


\section{REFERENCES}

Achtemeier SD, Morris LV, Finnegan CL. 2003. Considerations for developing evaluations of online courses. Journal of Asynchronous Learning Networks 7. URL: http://www.aln.org/publications/jaln/v7nl/v7nl_achtemeier.asp.

Anderson RH, Brown NA, Moorman AF. 2006. Development and structures of the venous pole of the heart. Dev Dyn 235:2-9.

Anderson RH, Brown NA, Webb S. 2002. Development and structure of the atrial septum. Heart 88:104-110.

Anderson RH, Webb S, Brown NA, Lamers W, Moorman A. 2003. Development of the heart: (2) Septation of the atriums and ventricles. Heart 89:949-958.

Berlage T. 1998. Augmented-reality communication for diagnostic tasks in cardiology. IEEE Trans Inf Technol Biomed 2:169-173.

Brune RM, Bard JB, Dubreuil C, Guest E, Hill W, Kaufman M, Stark M, Davidson D, Baldock RA. 1999. A three-dimensional model of the mouse at embryonic day 9. Dev Biol 216:457-468.

Dev P. 1999. Imaging and visualization in medical education. IEEE Comp. Graph. \& Appl. 19:21-31.

Dhenain M, Ruffins SW, Jacobs RE. 2001. Three-dimensional digital mouse atlas using highresolution MRI. Dev Biol 232:458-470.

Harvey RP, Rosenthal N. 1999. Heart development. In. San Diego: Academic Press. p 159189.

Higgins WE, Ramaswamy K, Swift RD, McLennan G, Hoffman EA. 1998. Virtual bronchoscopy for three-dimensional pulmonary image assessment: state of the art and future needs. Radiographics 18:761-778. 
His W. 1880. Die Area interposita, die Eustachische Klappe und die Spina vestibuli. In: Anatomie menschlicher Embryonen. Leipzig: FCW Vogel. p 49-152.

Houyel L. 1998. Embryologie cardiaque. Paris: Elsevier.

Huang C. 2005. Designing high-quality interactive multimedia learning modules. Comput Med Imaging Graph 29:223-233.

Kim JS, Viragh S, Moorman AF, Anderson RH, Lamers WH. 2001. Development of the myocardium of the atrioventricular canal and the vestibular spine in the human heart. Circ Res 88:395-402.

Laan AC, Lamers WH, Huijsmans DP, Te Kortschot A, Smith J, Strackee J, Los JA. 1989. Deformation-corrected computer-aided three-dimensional reconstruction of immunohistochemically stained organs: application to the rat heart during early organogenesis. Anat Rec 224:443-457.

Larsen WJ. 1993. Human embryology. In, 1rst ed. New York: Churchill Livingston Inc. p $131-165$.

Ringwald M, Baldock R, Bard J, Kaufman M, Eppig JT, Richardson JE, Nadeau JH, Davidson D. 1994. A database for mouse development. Science 265:2033-2034.

Röse C. 1899. Zur Entwicklungsgeschichte des Saugertierherzens. Morphol Jahr 15:436-456.

Schleich J-M, Dillenseger J-L, Andru S, Almange C, Coatrieux J-L. 2002. Understanding normal cardiac development using animated models. IEEE Comp. Graph. \& Appl. 22:14-19.

Schleich JM, Almange C. 2000. Four-dimensional virtual pictures: a new method for teaching cardiac embryology. In: 49th Annual Scientific Session of American College of Cardiology: J. Am. Coll. Cardiol. p 548 A.

Schoenwolf GC, Bleyl SB, Brauer PR, Francis-West PH. 2008. Larsen's human embryology, 4th ed: Churchill Livingstone. 
Sharpe J, Ahlgren U, Perry P, Hill B, Ross A, Hecksher-Sorensen J, Baldock R, Davidson D. 2002. Optical projection tomography as a tool for 3D microscopy and gene expression studies. Science 296:541-545.

Snarr BS, Kern CB, Wessels A. 2008. Origin and fate of cardiac mesenchyme. Dev Dyn 237:2804-2819.

Snarr BS, O'Neal JL, Chintalapudi MR, Wirrig EE, Phelps AL, Kubalak SW, Wessels A. 2007a. Isl1 expression at the venous pole identifies a novel role for the second heart field in cardiac development. Circ Res 101:971-974.

Snarr BS, Wirrig EE, Phelps AL, Trusk TC, Wessels A. 2007b. A spatiotemporal evaluation of the contribution of the dorsal mesenchymal protrusion to cardiac development. Dev Dyn 236:1287-1294.

Webb S, Brown NA, Anderson RH. 1996. The structure of the mouse heart in late fetal stages. Anat Embryol (Berl) 194:37-47.

Weninger WJ, Mohun T. 2002. Phenotyping transgenic embryos: a rapid 3-D screening method based on episcopic fluorescence image capturing. Nat Genet 30:59-65.

Wenink AC, Chon Y. 1984. The value of graphic reconstructions-comparison with scanning electron microscopy. Anat Rec 210:537-540.

Wessels A, Anderson RH, Markwald RR, Webb S, Brown NA, Viragh S, Moorman AF, Lamers WH. 2000. Atrial development in the human heart: an immunohistochemical study with emphasis on the role of mesenchymal tissues. Anat Rec 259:288-300.

Whiten S, Smart SD, McLachlan JC, Aiton JF. 1998. Computer-aided interactive threedimensional reconstruction of the embryonic human heart. J Anat 193:337-345. 
APPENDIX: Evaluation sheet

Did you use the animation?

Often $\quad$ Moderately $\quad$ Seldom $\quad$ Never $\quad$ Not Applicable

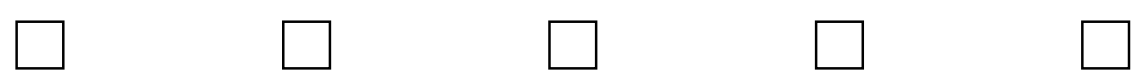

When did you use the animation?

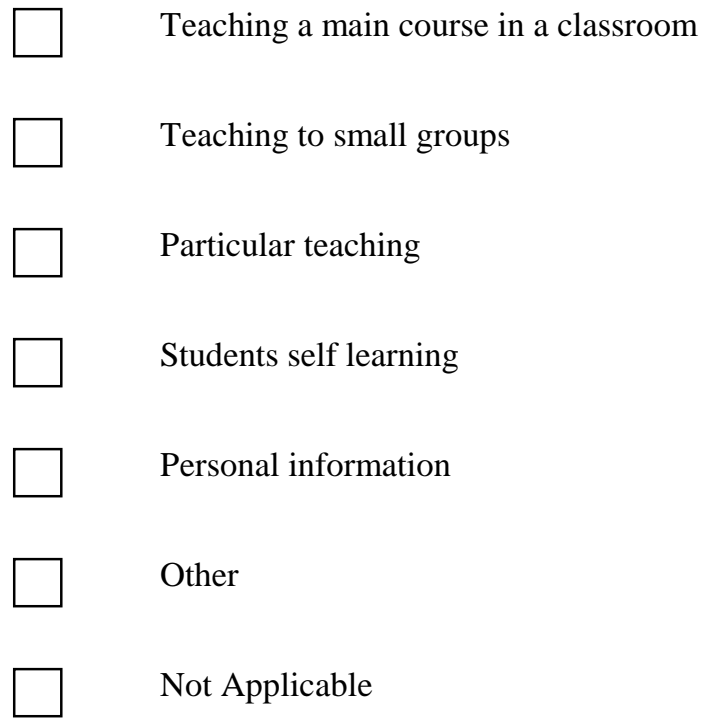

How many times by year do you use the animation?

$>5$

$2-5$

1

Never
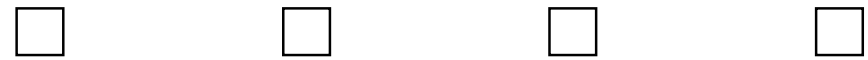

Is the animation available for the students?

Yes

No
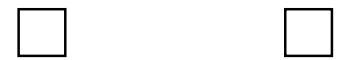

Navigation through the modules is easy.

Strongly Agree

Agree

Neither Agree

Disagree

nor Disagree
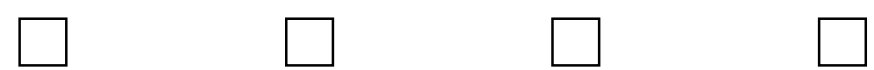
The 3D sequence module was useful for understanding cardiac embryology

Strongly Agree
Neither Agree

nor Disagree

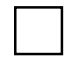

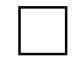

Disagree

Strongly

Disagree
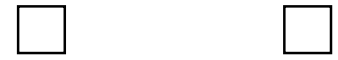

Not Applicable

The animation helped with conceptual understanding.

Strongly Agree

Agree

Neither Agree

Disagree

Strongly

Not Applicable

nor Disagree

Disagree
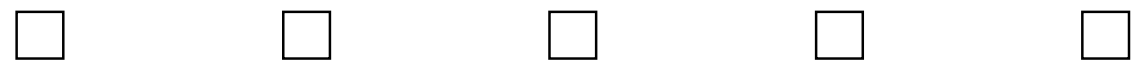

How do you feel the students learned the concepts with the animation?

What are there main feedbacks?

Did you encounter technical difficulties and did you have suggestions for improvements? 


\section{FIGURES}

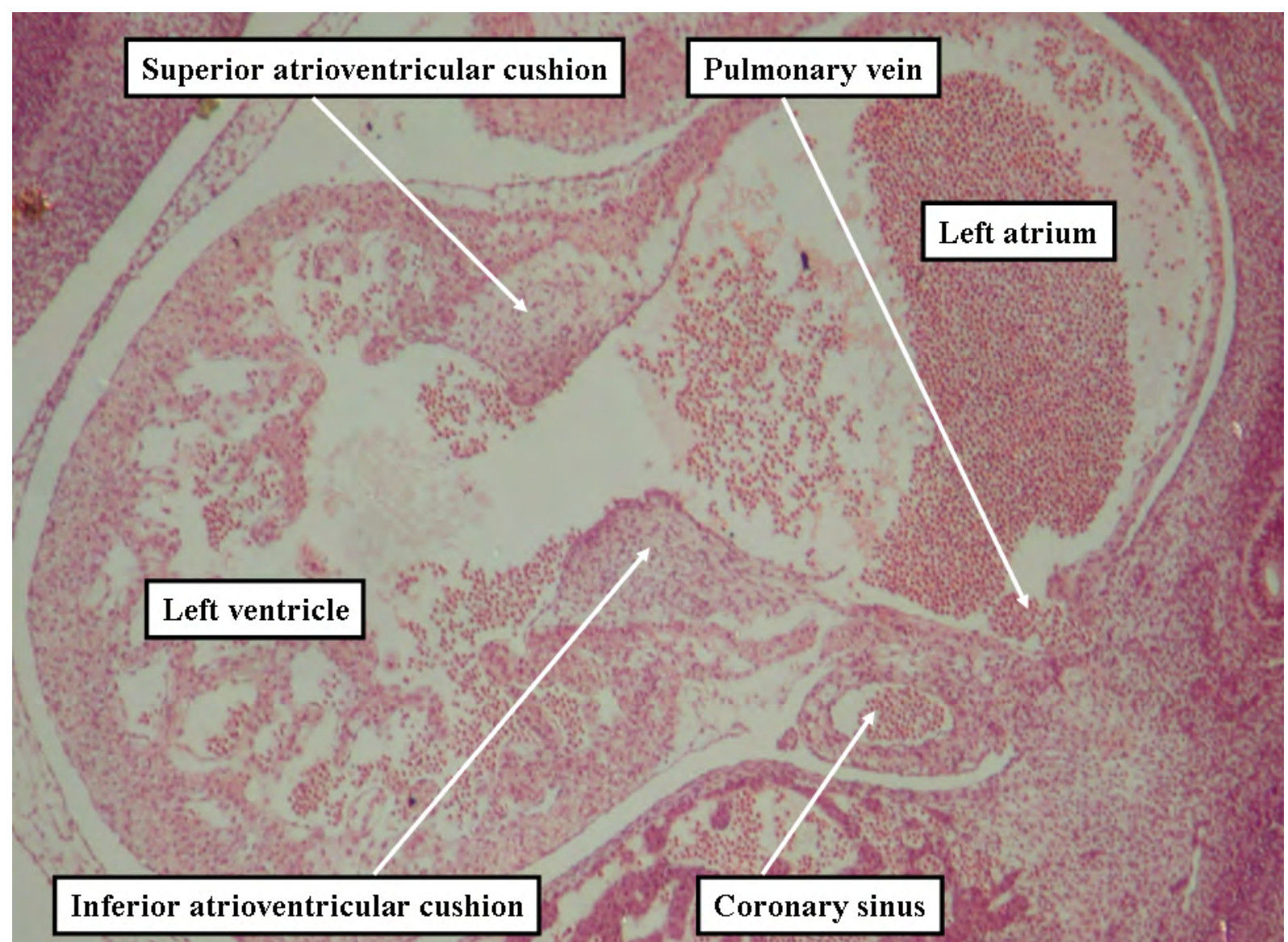

Figure 1. This sagittal section is from a human embryo of Carnegie stage 14, taken in a plane paralleling the so-called parasternal long axis section. It shows the initial location of the solitary pulmonary vein adjacent to the atrioventricular junction. Note the unfused cushions in the atrioventricular canal. 


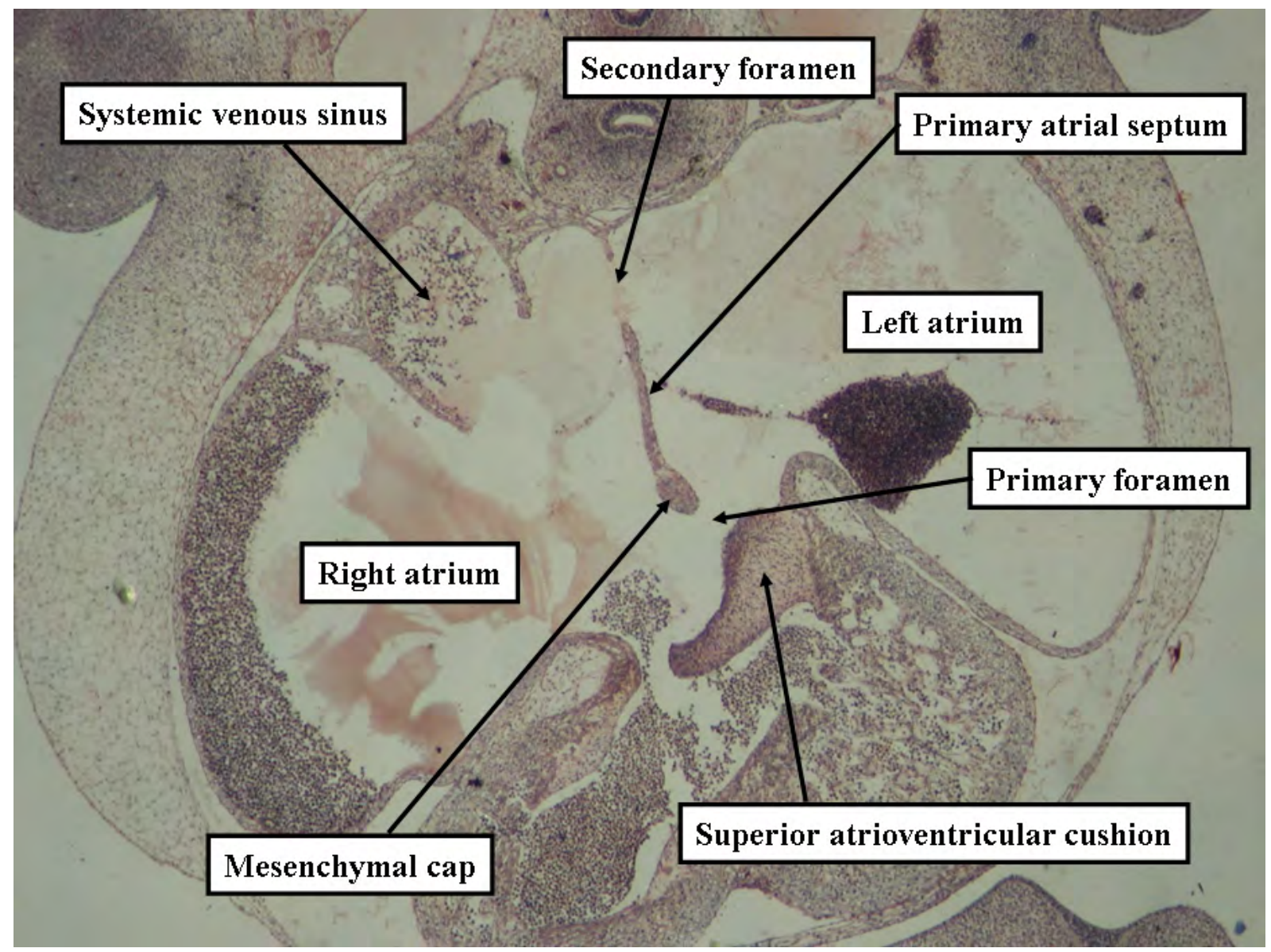

Figure 2. This frontal section, again from a human embryo, but at the early part of Carnegie stage 16, shows the primary atrial septum, with its mesenchymal cap, growing towards the superior endocardial cushion. 


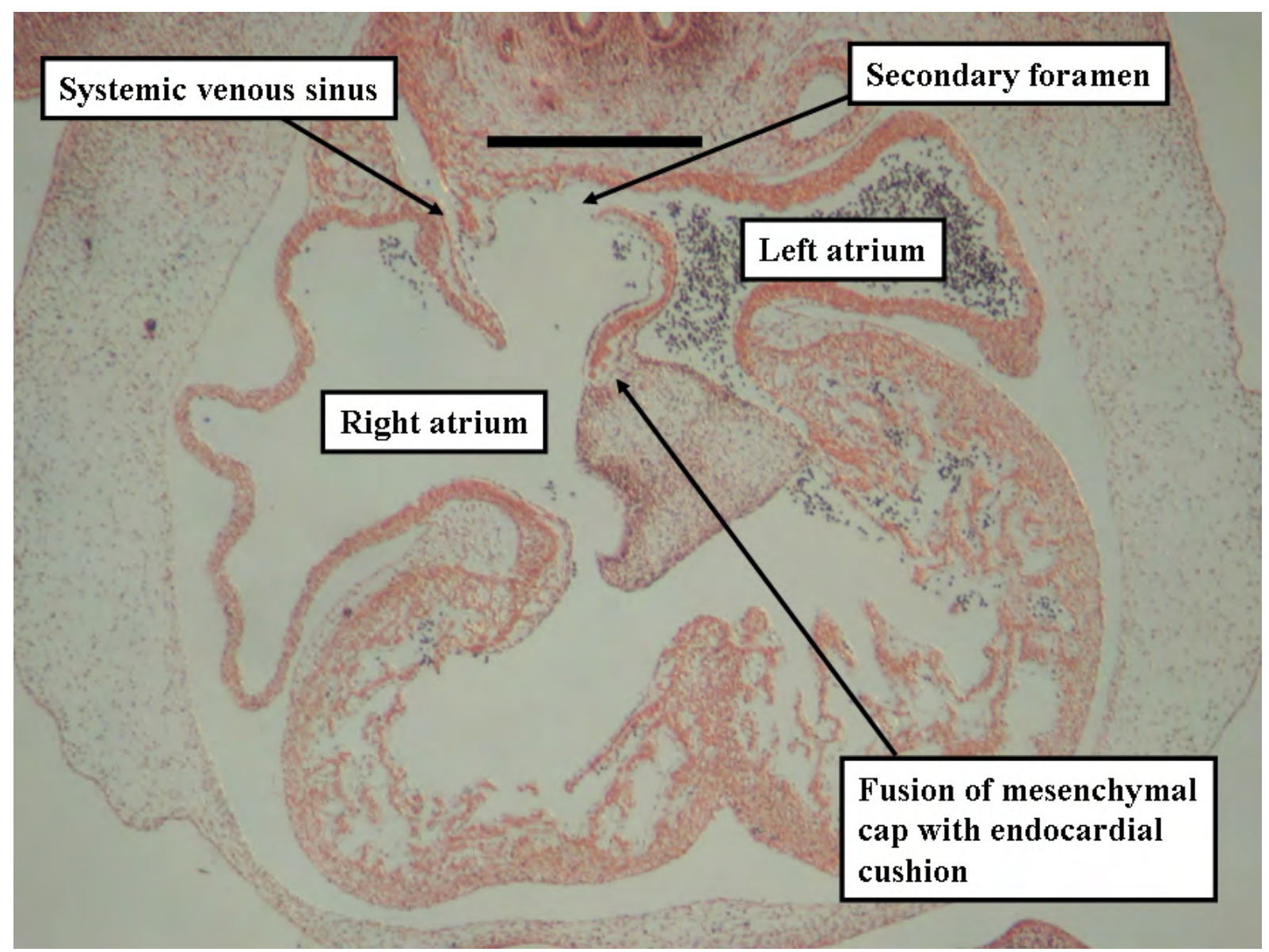

Figure 3. This section, again in the frontal plane, is from a human embryo in the later part of the $16^{\text {th }}$ Carnegie stage. The mesenchymal structures have fused, but the superior roof of the atrial component is flat (black line), with no evidence of formation of a "septum secundum". 


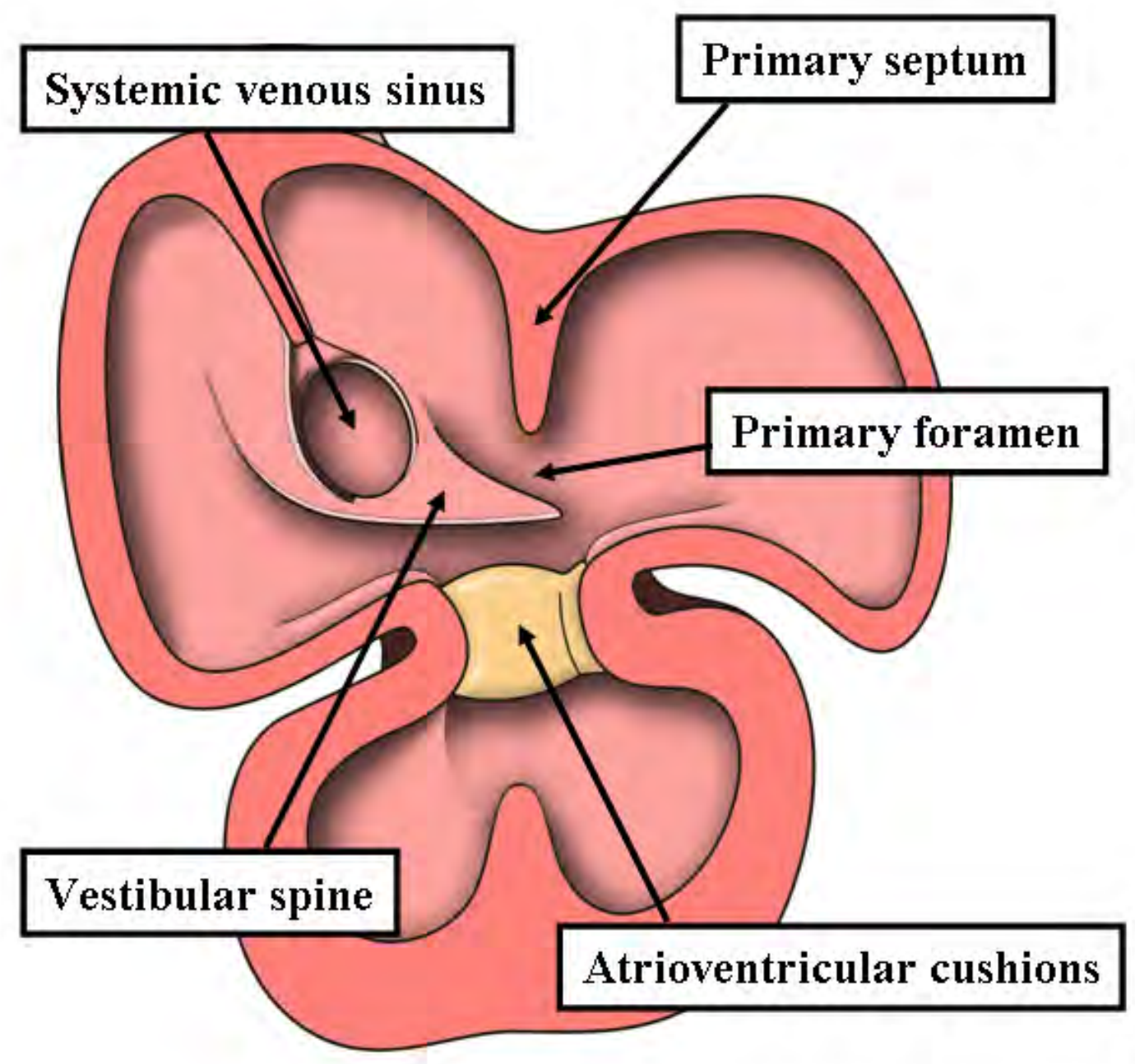

Figure 4. The cartoon shows a representation of the reconstruction made by His in the $19^{\text {th }}$ century to show the development of the "spina vestibule", or vestibular spine. 


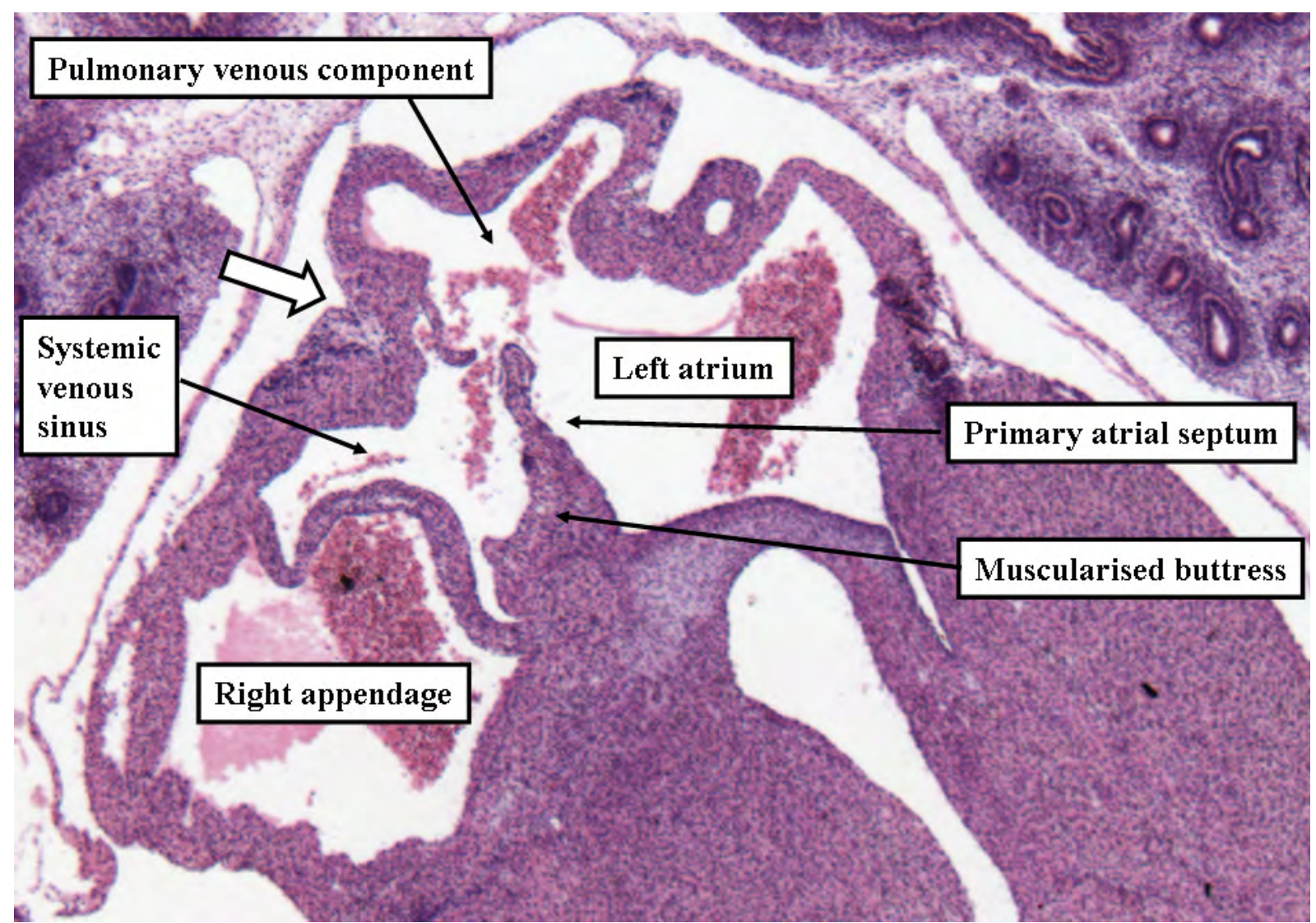

Figure 5. This section comes from a human embryo after the completion of cardiac septation.

It shows that formation of the superior interatrial fold is not possible until the pulmonary veins have migrated to the atrial roof. Note the muscularisation of the mesenchymal mass to form the antero-inferior buttress of the atrial septum. 


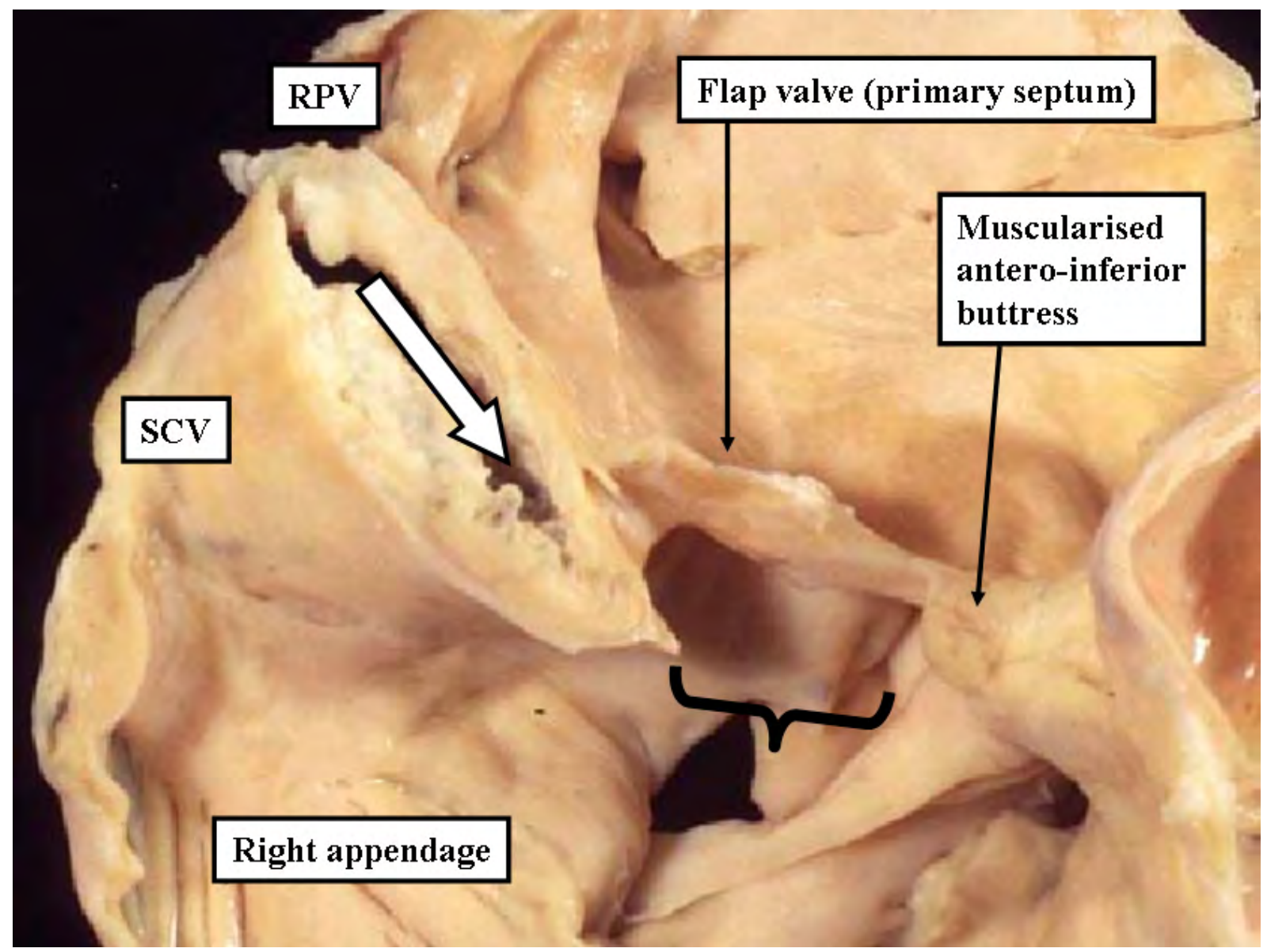

Figure 6. This section is from an adult human heart, and shows that the so-called "septum secundum" is, in reality, a deep interatrial fold. It also shows the muscularised antero-inferior buttress. 

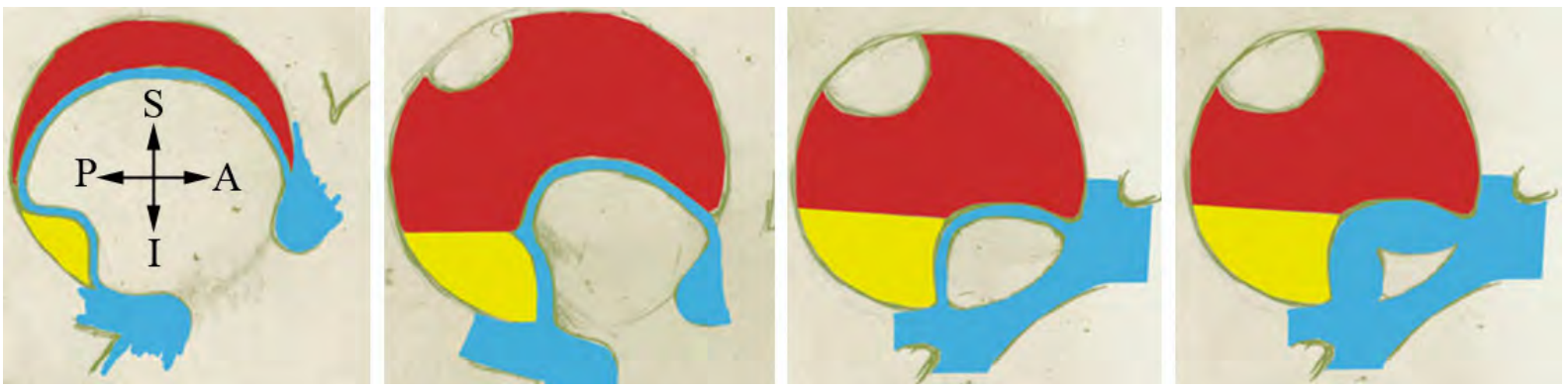

Figure 7: Drawing of the storyboard in colour: The origins, forms, dimensions, spatial extension and the relationship in space and temporal changes between the different elements involved in the atrial septation are defined. In red: the primary atrial septum; in yellow: the vestibular spine; in blue: the atrioventricular endocardial cushions and the mesenchymal cap of the primary atrial septum and the mesenchymal tissue of the vestibular spine. A: anterior, $\mathrm{P}$ : posterior, S: superior, I: inferior.

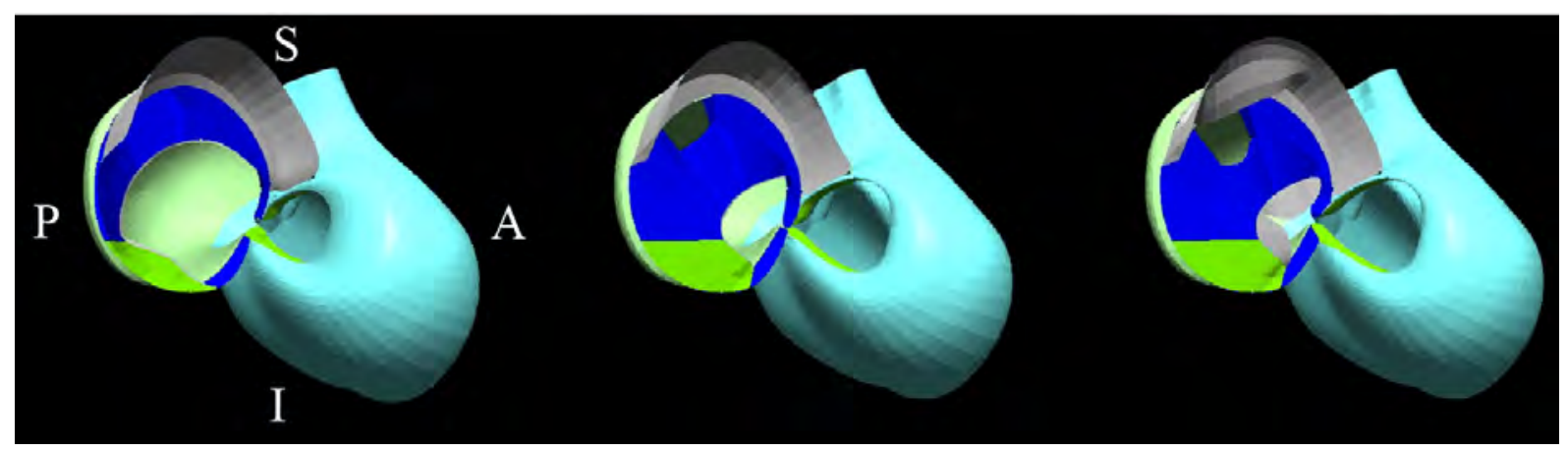

Figure 8: Successive pictures showing the deformations of the shapes over time. A: anterior, P: posterior, S: superior, I: inferior. 

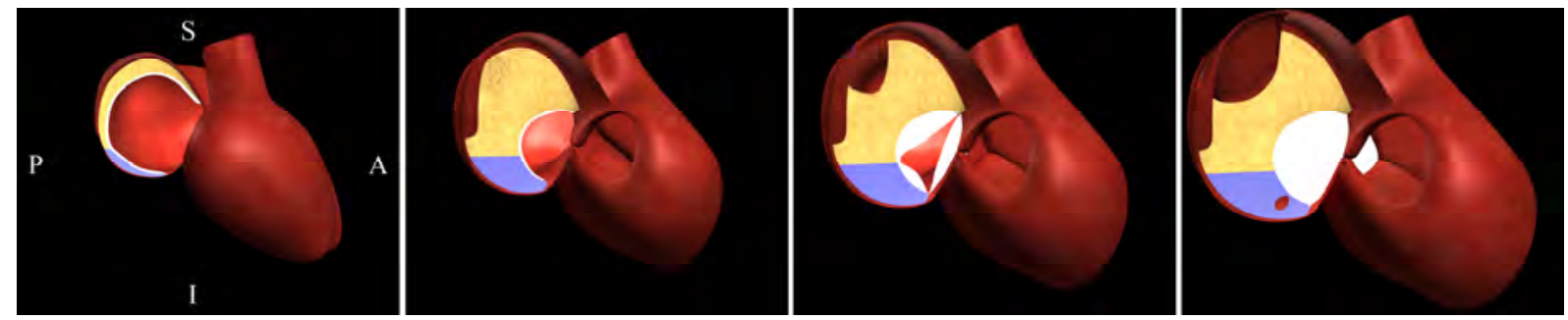

Figure 9: The hypothesis used to explain atrial septation is designed as follows: the primary septum (in yellow) grows from the cranial part of the primary atrium, leaving a foramen distal to its leading edge called the primary foramen. The vestibular spine (in purple) enters the heart and grows towards the inferior atrioventricular cushion on the right side of the primary septum. A second partition, in brown, is produced by folding of the atrial roof. The primary foramen is closed by an expansion of tissue coming from the endocardial cushions, in white. The successive pictures are particularly relevant, permitting students to visualize the chronological and spatial changes that occur within the atrial chambers. A: anterior, $\mathrm{P}$ : posterior, S: superior, I: inferior. 